\title{
Evaluación de impacto ambiental en el sector de la construcción de viviendas: Caso estudio viviendas del MIDUVI en el cantón "La Maná", Cotopaxi, Ecuador
}

\author{
Environmental impact assessment in the housing construction \\ sector: Case study of MIDUVI homes in the canton "La Maná", \\ Cotopaxi, Ecuador
}

\author{
Víctor Paul Carvajal Padilla ${ }^{1}$, Robinson Rigoberto Ambuludi Paredes ${ }^{1}$, Heidi Chávez ${ }^{1}$, Estalin Grefa ${ }^{1}$, Karel Diéguez Santana ${ }^{1 *}$ \\ ${ }^{1}$ Facultad Ciencias de la Vida, Carrera Ingeniería Ambienta, Universidad Estatal Amazónica, Paso lateral km 2 1/2 vía Tena. \\ Puyo, Pastaza, Ecuador
}

\begin{abstract}
Resumen La industria de la construcción es una de las principales causantes de impactos ambientales y daños a la biodiversidad a nivel mundial. El objetivo de este estudio es realizar una evaluación de impacto ambiental en la construcción de viviendas de MIDUVI del sector "La Soledad" en el Cantón La Maná, Ecuador. Se identificó la línea base y se describieron las actividades del proyecto. Para identificar los impactos ambientales que se pueden presentar en los componentes físicos, biótico y socioeconómicocultural se aplicó la metodología de la matriz modificada de Leopold. Los resultados del estudio revelan que la modificación del hábitat, la remoción de cobertura vegetal y la urbanización son los impactos ambientales más significativos dentro del área de estudio. Además, se puede mencionar que el reciclaje de residuos es una de las actividades positivas dentro de la construcción, por lo tanto, se deberán formular planes de manejo ambiental amigables para dar seguimiento y control en las primeras etapas de construcción.
\end{abstract}

Palabras clave Calidad ambiental, estudio de impacto ambiental, factores ambientales.

\begin{abstract}
The construction industry is one of the main causes of environmental impacts and damage to biodiversity worldwide. The objective of this study is to carry out an environmental impact assessment in the construction of MIDUVI housing in the "La Soledad" area, La Maná Canton, Ecuador. The baseline was identified, and the project activities were described. To identify the environmental impacts that may occur in the physical, biotic and socioeconomic-cultural components, the methodology of Leopold's modified matrix was applied. The study results reveal that habitat modification, vegetation cover removal and urbanization are the most significant environmental impacts within the study area. In addition, it can be mentioned that waste recycling is one of the positive activities within construction, therefore, friendly environmental management plans should be formulated to follow up and control in the early stages of construction.
\end{abstract}

Keywords Environmental quality, environmental impact study, environmental factors.

* Corresponding author: karel.dieguez.santana@gmail.com

\section{Introducción}

La industria de la construcción provoca grandes impactos en el medio ambiente y la biodiversidad a través del uso de recursos y las emisiones atmosféricas [1]. Según las estadísticas del Programa de las Naciones Unidas para el Medio Ambiente (PNUMA en español y UNEP en inglés), la construcción de edificaciones representa el $4 \%$ del uso mundial de energía, el 30\% de las emisiones de gases de efecto invernadero relacionadas con la energía, aproximadamente el $12 \%$ del uso del agua y casi el $40 \%$ de los residuos que se producen a nivel mundial [2].
Adicionalmente, el sector de la construcción es el principal consumidor de materias primas y según Dixit, et al. [3], es responsable del agotamiento del $40 \%$ de la piedra, grava y arena; $25 \%$ de madera; y $16 \%$ de agua dulce.

Las diferentes actividades que se generan en este sector pueden indicar distintos impactos ambientales, por lo que la capacidad de evaluar y comparar los impactos ambientales de diferentes actividades de construcción, pueden ayudar al proceso de minimizar las emisiones en diferentes procesos de construcción de edificaciones [4]. Además, como plantean Enshassi, et al. [5] la identificación de los impactos de los proyectos de construcción sobre el entorno es una tarea que 
debe ser tratada, con el fin de poder realizar una protección eficaz bajo una evaluación de impacto ambiental.

Los estudios de impacto ambiental en el Ecuador han sido de vital importancia para la mayoría de los macro proyectos a nivel nacional y contribuyen a la reducción de los problemas ambientales, que genera todo proyecto, obra o actividad. Este proceso permite evaluar el proyecto desde el punto de vista ambiental y generar alternativas de procedimientos para alcanzar un desarrollo sustentable [6].

El Cantón La Maná, perteneciente a la provincia de Cotopaxi, se encuentra en el centro Sur del Ecuador a unos $240 \mathrm{~km}$ de la ciudad de Quito. Es una de las principales entradas a la costa Ecuatorial, es un punto de intercambio entre productores agrícolas tanto de la sierra como de la costa, y al tener un gran auge económico, su población ha empezado a crecer durante los últimos años [7]. Al 2021, soñamos un Ecuador donde todos tengamos vivienda digna, especialmente los más pobres, donde los niños y las niñas no sufran de desnutrición, y los jóvenes puedan acceder a la educación superior, donde no exista violencia contra las mujeres, y se garanticen los derechos de la naturaleza

Según la Secretaria Nacional de Planificación y Desarrollo [8] existe un déficit de vivienda a nivel nacional y es un deber gubernamental prioritario de erradicar la pobreza y promover el progreso económico, social y cultural del país, por lo que se ha diseñado un proyecto de crecimiento del área inmobiliaria, orientado a atender las necesidades de una población rural emergente que poco a poco intenta romper la frontera entre los campos y las grandes urbes.

El Ministerio de Desarrollo Urbano y Vivienda (MIDUVI), emprendió la construcción de viviendas llamado "La Soledad" que se dedicará a la creación de 31 viviendas para 31 familias, presentes en la parroquia La Maná de origen rural que se encuentran dentro del Cantón del mismo nombre. El fin de este proyecto es reducir en un $12 \%$ el déficit de vivienda en El Cantón. Sin embargo, debido a que el lugar de implementación del presente proyecto se centra en un área donde la actividad agrícola extensiva ocupa la mayor parte de los terrenos a ser intervenidos, se ha decidido efectuar una evaluación de impacto ambiental para reducir eventualidades ambientales que se generen durante la ejecución del proyecto.

Por otra parte, las matrices de valoración se han convertido en un método sencillo, para identificar los impactos ambientales de diferentes actividades obras o proyectos. Estos criterios metodológicos han sido empleados en estudios anteriores para la identificación y valoración de impactos en actividades productivas, cultivo de la pitahaya [9], operación y funcionamiento del camales [10] o en obras constructivas, como la construcción de plantas de tratamiento de aguas residuales [11].

El objetivo de este estudio fue analizar los impactos ambientales de la construcción de lotes de edificaciones del MIDUVI en La Maná, y así poder identificar el grado de perturbación que puede generar hacia el medio físico y ecológico circundante al área de influencia de la obra y de esta manera establecer medidas ambientales que permitan prevenir, controlar, minimizar y mitigar los posibles impactos generados dentro del proyecto.

\section{Materiales y métodos}

\subsection{Localización}

El estudio se realizó en la provincia de Cotopaxi, parroquia La Maná, Cantón La Maná, en las coordenadas 17 M 693802.16 m E $9893557.45 \mathrm{~m} \mathrm{~S}$, a una altitud de 170 msnm. Su temperatura promedio es de $22{ }^{\circ} \mathrm{C}$ y su precipitación de $2694 \mathrm{~mm}$ al año (Ver la figura 1).

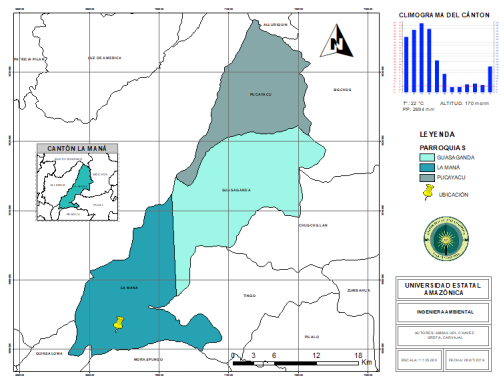

Figura 1. Mapa de ubicación del área de estudio, Cantón y parroquias.

\subsection{Descripción de la actividad}

Se realizó el estudio de impacto ambiental, sobre la actividad de construcción de viviendas del MIDUVI (Ministerio de Desarrollo Urbano y Vivienda), con el fin de tener un panorama más específico de todos los impactos, tanto positivos como negativos, que genera este tipo de proyectos. Se detallan todos los procesos llevados a cabo dentro de la obra, fueron tomados en consideración al momento de realizar el presente estudio, haciendo énfasis en los más significativos que son: remoción de suelo, cobertura vegetal, disposición final de desechos y etapa de construcción (cimentación debido a la generación de material particulado y polvorines que afectarían directamente a la salud de los trabajadores) (figura 2).

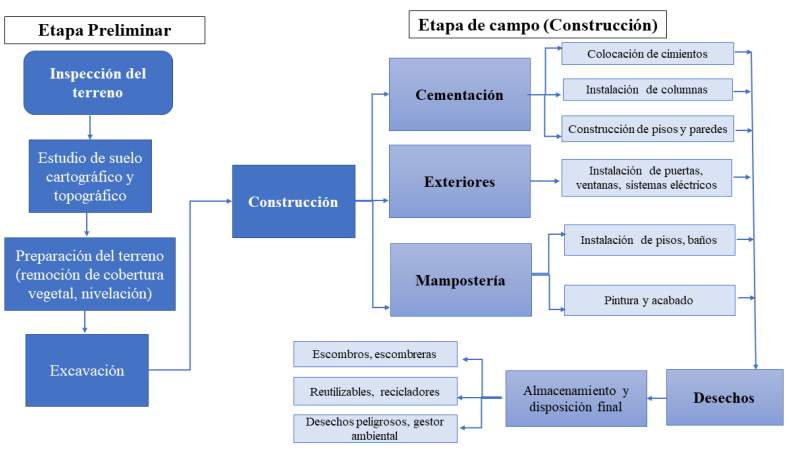

Figura 2. Diagrama de flujo de las operaciones y actividades de la construcción. 


\subsection{Levantamiento de información de la línea base}

En esta etapa se realizaron varias visitas de campo y una búsqueda exhaustiva en medios bibliográficos, con el objetivo de obtener información confiable que nos permita caracterizar el medio en el cual se desarrollan las actividades de construcción. En el caso de las visitas de campo, estas fueron para examinar mediante observación directa, los diferentes componentes, físico, biótico y socioeconómico cultural, mientras que en los casos donde no fue posible obtener información directa, se consultaron fuentes bibliográficas primarias y secundarias, reportes, informe ambiental y estadístico del cantón, INEC, MAG, entre otros.

\subsection{Métodos de evaluación}

En la actualidad existen un gran número de métodos de evaluación de impactos ambientales, teniendo en consideración que varios han sido creados para un tema específico y que difícilmente se lograrían acoplar a temas generales [10]. Los sistemas de matrices son ampliamente conocidos. Para el presente estudio se empleó la matriz de Leopold modificada. Esta es una matriz de valoración en la cual se cuantifican los impactos a partir de la magnitud e importancia. Este método permite unir los factores ambientales a ser afectados y las acciones humanas que producen esa afectación, con el fin de medir las interacciones, tanto negativas como positivas y de esta manera valorar cuantitativamente la magnitud y el impacto que generan las actividades. Los resultados de la aplicación de esta metodología permiten formular recomendaciones de mejora o establecer un plan de manejo ambiental, en caso de ser necesario [11].

El valor del impacto se obtuvo a partir de la ecuación 1, que está en dependencia de la magnitud e importancia. De esta ecuación, los resultados positivos, significa que el impacto será positivo, y si el valor del impacto fuese negativo, el impacto final será negativo.

$$
\text { Impacto }=\text { Importancia } \times \text { Magnitud }
$$

En el caso de la magnitud, describe el grado de afección que podría tener un factor ambiental. Se empleó un rango de 1, para bajas hasta 10 para muy altas, mientras que el caso de la importancia, se consideraron seis tipos distintos de variables con relevancia específica:

Naturaleza: Define si un impacto es positivo o negativo.

Probabilidad: Define cual es la probabilidad de ocurrencia de un impacto.

Duración: Define la línea temporal de un impacto ambiental.

Reversibilidad: Define si el impacto se podrá remediar a corto o largo plazo.
Intensidad: Define el grado de afectación que posiblemente tendrá un impacto ambiental.

Extensión: Define el grado de diseminación del impacto ambiental.

En este caso, se obtuvo a partir de la ecuación 2, y los valores de las variables se seleccionaron basado en los criterios de la tabla 1 .

$$
\begin{aligned}
& \text { Magnitud }=\text { Naturaleza } \times \text { Probabilidad } \times(\text { Duración } \\
& \text { Reversibilidad }+ \text { Intensidad }=\text { Extensión })
\end{aligned}
$$

Tabla 1. Rangos numéricos utilizados para las variables Naturaleza, Probabilidad, Duración, Reversibilidad, Intensidad y Extensión

\begin{tabular}{|l|l|l|l|l|l|}
\hline Naturaleza & Probabilidad & Duración & Reversibilidad & Intensidad & Extensión \\
\hline $\begin{array}{l}\text { Positiva } \\
+1\end{array}=$ & $\begin{array}{c}\text { Poco Probable } \\
=0,1\end{array}$ & Temporal=1 & Acorto plazo=1 & Baja =1 & Puntual=1 \\
\hline $\begin{array}{l}\text { Negativa }=- \\
1\end{array}$ & Probable $=0,5$ & $\begin{array}{c}\text { Permanente } \\
=2\end{array}$ & A largo plazo=2 & Media =2 & Local =2 \\
\hline- & Cierto $=1$ & - & & Alta $=3$ & $\begin{array}{l}\text { Regional }= \\
3\end{array}$ \\
\hline
\end{tabular}

Finalmente, los valores de impacto obtenidos se categorizaron empleando los criterios de la tabla 2.

Tabla 2. Rangos numéricos utilizados para la caracterización del impacto ambiental

\begin{tabular}{|c|c|c|}
\hline Rango & Característica & Significancia \\
\hline 80 a 100 & E+ & Muy significativo positivo \\
\hline 60 a 80 & D+ & Significativo positivo \\
\hline 41 a 60 & C+ & $\begin{array}{c}\text { Medianamente significativo } \\
\text { positivo }\end{array}$ \\
\hline 21 a 40 & B+ & Poco significativo positivo \\
\hline 0 a 20 & A+ & No significativo positivo \\
\hline 0 a -20 & a- & No significativo negativo \\
\hline-21 a -40 & b- & Poco significativo negativo \\
\hline-41 a - 60 & c- & $\begin{array}{c}\text { Medianamente significativo } \\
\text { negativo }\end{array}$ \\
\hline-61 a - 80 & d- & Significativo negativo \\
\hline-81 a -100 & e- & Muy significativo negativo \\
\hline
\end{tabular}

\subsection{Plan de manejo ambiental}

Una vez identificados los impactos ambientales es necesario proponer medidas que permitan mitigar y gestionar los efectos ambientales de la actividad, obra o proyecto [10]. En Ecuador, el Reglamento al Código Orgánico del Ambiente (Decreto Ejecutivo $\mathrm{N}^{\circ} 752$, Registro Oficial Suplemento $\mathrm{N}^{\circ}$ 507 del 19 de junio de 2019), en el artículo 435, menciona cuales son los sub-planes de manejo ambiental a considerar los aspectos ambientales, impactos y riesgos identificados en proyecto, obra o actividad en análisis. La tabla 3, muestra una descripción breve de cada uno de los planes seleccionados y 
Diéguez (et al): Evaluación de impacto ambiental en el sector de la construcción de viviendas: Caso estudio viviendas del MIDUVI en el cantón “La Maná”, Cotopaxi, Ecuador

las debidas justificaciones de los planes no considerados en este estudio.

Tabla 3. Descripción de planes de manejo ambiental utilizados

\begin{tabular}{|c|c|}
\hline Plan de manejo & Descripción \\
\hline $\begin{array}{lr}\text { Plan } & \text { de } \\
\text { prevención } & y \\
\text { mitigación } & \text { de } \\
\text { impactos } & \end{array}$ & $\begin{array}{l}\text { Plan incluido para la mitigación de } \\
\text { daños ocasionados por el área de } \\
\text { influencia del presente proyecto. }\end{array}$ \\
\hline $\begin{array}{ll}\text { Plan de } & \text { dentingencias }\end{array}$ & $\begin{array}{l}\text { Plan no incluido debido a que la } \\
\text { mayor parte de las contingencias y } \\
\text { recomendaciones se encuentran } \\
\text { presentes en el plan de seguridad y } \\
\text { salud ocupacional. }\end{array}$ \\
\hline $\begin{array}{l}\text { Plan de manejo } \\
\text { de desechos }\end{array}$ & $\begin{array}{l}\text { Plan incluido para controlar la } \\
\text { generación y eliminación de residuos } \\
\text { generados en el momento de la } \\
\text { operación, cierre y abandono. }\end{array}$ \\
\hline $\begin{array}{l}\text { Plan de relaciones } \\
\text { comunitarias }\end{array}$ & $\begin{array}{l}\text { Plan no incluido, debido a que las } \\
\text { relaciones comunitarias estuvieron a } \\
\text { cargo del MIDUVI. }\end{array}$ \\
\hline $\begin{array}{l}\text { Plan de } \\
\text { rehabilitación de } \\
\text { áreas afectadas }\end{array}$ & $\begin{array}{l}\text { Plan implementado, para la } \\
\text { recuperación del suelo, factor } \\
\text { ambiental importante en el presente } \\
\text { proyecto }\end{array}$ \\
\hline $\begin{array}{l}\text { Plan de rescate de } \\
\text { vida silvestre, de } \\
\text { ser aplicable }\end{array}$ & $\begin{array}{l}\text { Plan no aplicable debido a que la } \\
\text { mayor parte del área de influencia es } \\
\text { utilizado para la agricultura. }\end{array}$ \\
\hline $\begin{array}{l}\text { Plan de seguridad } \\
\text { y salud } \\
\text { ocupacional }\end{array}$ & $\begin{array}{l}\text { Plan incluido, dada las circunstancias } \\
\text { a la que los trabajadores del ámbito de } \\
\text { la construcción están expuestos. } \\
\text { Nota: Este plan actualmente no se } \\
\text { encuentra estipulado por el Art. } 435 \text {, } \\
\text { sin embargo, es de vital importancia } \\
\text { para el presente proyecto y, por ende, } \\
\text { es utilizado en esta metodología. }\end{array}$ \\
\hline $\begin{array}{l}\text { Plan de cierre y } \\
\text { abandono }\end{array}$ & $\begin{array}{l}\text { Plan incluido en el proyecto dado la } \\
\text { naturaleza de la actividad es esencial } \\
\text { para un abandono sin } \\
\text { desconformidades. }\end{array}$ \\
\hline & Paisajismo, suelo, aspectos culturales \\
\hline
\end{tabular}

\section{Resultados y discusión}

\subsection{Diagnóstico ambiental (línea base)}

La construcción de viviendas ubicada en la parroquia $\mathrm{La}$ Maná, Cantón La Maná, provincia de Cotopaxi, lugar de estudio es una zona rural en la cual la presencia de caseríos es escasa porque los suelos en su mayor parte se encuentran ocupados por fincas agrícolas y ganaderas, razón por la cual la presencia de vegetación nativa es escasa y se limita a sitios de difícil acceso, factores que inciden para la baja presencia de fauna silvestre. La tabla 4 , muestra los principales resultados de la descripción de la línea base.

Tabla 4. Descripción de la línea base utilizados actualmente

\begin{tabular}{|c|c|}
\hline $\begin{array}{c}\text { Componente } \\
\text { ambiental }\end{array}$ & Estado actual \\
\hline
\end{tabular}

Medio Físico

Clima

El sitio en estudio tiene un clima Tropical Megatérmico Húmedo que abarca

1880,56 hectáreas, que equivalen al $31,3 \%$ de la superficie.

\begin{tabular}{|l|l|}
\hline Temperatura & $\begin{array}{l}\text { Las temperaturas fluctúan entre } 17-32^{\circ} \mathrm{C}, \\
\text { promedio de } 24^{\circ} \mathrm{C} \text {, durante el periodo } \\
2003-2012 .\end{array}$ \\
\hline Hidrología & $\begin{array}{l}\text { Los caudales en los últimos } 10 \text { años } \\
\text { varían entre } 92,4 \mathrm{~m} 3 / \mathrm{s} \text { hasta } 308,5 \mathrm{~m} 3 / \mathrm{s} .\end{array}$ \\
\hline Tipo de suelo & $\begin{array}{l}\text { El área de estudio se ubica en el grupo de } \\
\text { suelos inceptisoles, los cuales ocupan una } \\
\text { superficie de } 400,031 \text { que corresponde al } \\
66,5 \% .\end{array}$ \\
\hline
\end{tabular}

Medio biótico

Flora

Se identificaron especies arbustivas, herbáceas espontáneas y pioneras que se han desarrollado por procesos dinámicos del ambiente y por procesos de realce natural.

\begin{tabular}{l|l}
\hline Fauna & $\begin{array}{l}\text { Ausencia total de especies faunísticas } \\
\text { nativas del lugar de estudio, debido a la } \\
\text { sobreexplotación en la zona. }\end{array}$
\end{tabular}

\section{Socioeconómico-Cultural}

\begin{tabular}{|c|c|}
\hline Cultivos & $\begin{array}{l}\text { La mayor parte los suelos han sido } \\
\text { ocupados por especies como banano } \\
\text { (Musa sapiens), yuca (Manihot } \\
\text { esculenta), café (Coffea arábica), cacao } \\
\text { (Teobroma cacao) y una variedad de } \\
\text { cítricos (naranja y mandarina). }\end{array}$ \\
\hline $\begin{array}{l}\text { Población } \\
\text { económicamente } \\
\text { activa }\end{array}$ & $\begin{array}{l}\text { (PEA) en el Cantón es del } 51,9 \% \text {, la } \\
\text { principal actividad es la agricultura con el } \\
55,1 \% \text {, seguida por el comercio con un } \\
12,5 \% \text {. }\end{array}$ \\
\hline $\begin{array}{l}\text { Acceso y usos del } \\
\text { agua }\end{array}$ & $\begin{array}{l}\text { El cantón cuenta con el } 84 \% \text { de servicio } \\
\text { de agua mediante red pública y el } 16 \% \\
\text { mediante el abastecimiento por vertientes } \\
\text { o pozos. }\end{array}$ \\
\hline Escolaridad & $\begin{array}{l}\text { El nivel de escolaridad en la población es } \\
\text { de } 7.5 \text { años para las mujeres y } 7.6 \text { años } \\
\text { para los hombres. }\end{array}$ \\
\hline
\end{tabular}

\subsection{Resultados de los instrumentos de valoración}

La tabla 5, muestra los principales impactos identificados entre las actividades y los componentes ambientales del área de estudio directa del proyecto. 
Diéguez (et al): Evaluación de impacto ambiental en el sector de la construcción de viviendas: Caso estudio viviendas del MIDUVI en el cantón “La Maná”, Cotopaxi, Ecuador

Tabla 5. Identificación de impactos según la matriz de Leopold modificada

\begin{tabular}{|c|c|c|c|c|c|c|c|c|c|c|}
\hline \multirow[b]{2}{*}{$\begin{array}{l}\text { Componentes } \\
\text { ambientales }\end{array}$} & \multicolumn{10}{|c|}{ Actividades } \\
\hline & 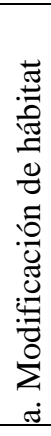 & 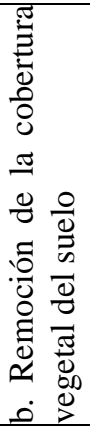 & 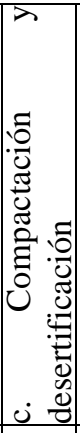 & 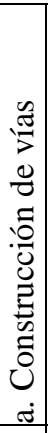 & 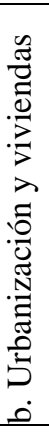 & 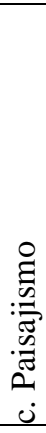 & 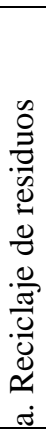 & 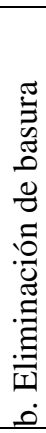 & 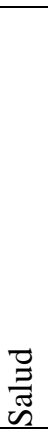 & 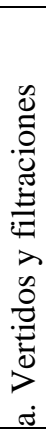 \\
\hline Suelo & $\mathrm{x}$ & $\mathrm{X}$ & $\mathrm{X}$ & $\mathrm{x}$ & & $\mathrm{x}$ & $\mathrm{x}$ & & & $\mathrm{x}$ \\
\hline Agua & $\mathrm{X}$ & & & & $\mathrm{x}$ & & $\mathrm{X}$ & & $\mathrm{X}$ & $\mathrm{X}$ \\
\hline Atmósfera & $\mathrm{X}$ & $\mathrm{X}$ & & $\mathrm{X}$ & & & $\mathrm{X}$ & & $\mathrm{X}$ & \\
\hline Flora & $\mathrm{x}$ & $\mathrm{x}$ & $\mathrm{X}$ & $\mathrm{x}$ & $\mathrm{x}$ & & $\mathrm{x}$ & & & \\
\hline Fauna & $\mathrm{X}$ & $\mathrm{x}$ & $\mathrm{X}$ & $\mathrm{X}$ & $\mathrm{X}$ & & $\mathrm{X}$ & & & \\
\hline $\begin{array}{l}\text { Interés } \\
\text { económico y } \\
\text { humano }\end{array}$ & & $\mathrm{X}$ & & $\mathrm{X}$ & $\mathrm{X}$ & & $\mathrm{X}$ & $\mathrm{X}$ & $\mathrm{X}$ & \\
\hline $\begin{array}{l}\begin{array}{l}\text { Aspectos } \\
\text { culturales }\end{array} \\
\end{array}$ & & $\mathrm{X}$ & & & $\mathrm{x}$ & $\mathrm{x}$ & & & & \\
\hline $\begin{array}{l}\text { Relaciones } \\
\text { ecológicas }\end{array}$ & $\mathrm{X}$ & $\mathrm{x}$ & $\mathrm{x}$ & & $\mathrm{x}$ & & $\mathrm{X}$ & & & \\
\hline
\end{tabular}

Por su parte, la tabla 6, muestra las valorizaciones de los impactos identificados. En los componentes ambientales, se puede apreciar que el medio físico se muestra muy afectado, principalmente el suelo (6 impactos negativos), atmósfera y agua (4 impactos cada uno). También, el medio biótico con flora y fauna, con 5 impactos negativos cada uno de ellos, respectivamente. Por otro lado, los principales impactos positivos se ubican en el componente socioeconómico, interés económico y humano (5 impactos positivos), pues la construcción de estas viviendas significa en mejora de las condiciones de vida de la población beneficiaria.

La construcción de viviendas genera un impacto negativo al ambiente, como lo es el deterioro de las características del suelo, aire y agua superficial que afecta directamente a la pérdida de especies nativas del lugar, mayor emisión de material particulado hacia la atmósfera, incluso llegando a generar la migración de especies, haciendo que abandonen su hábitat natural. Podemos observar que estas actividades que generan afecciones al ambiente están generando un aumento en las actividades económicas del lugar, de manera que existe mayor cantidad de fuentes de empleo, debido a que, para llevar a cabo las diferentes actividades dentro de la construcción, se necesita recurso humano para ejecutarlas y estas son remuneradas.
Tabla 6. Valoración de los impactos ambientales

\begin{tabular}{|c|c|c|c|c|c|c|c|c|c|c|}
\hline \multirow[b]{2}{*}{$\begin{array}{l}\text { Componentes } \\
\text { ambientales }\end{array}$} & \multicolumn{10}{|c|}{ Actividades } \\
\hline & 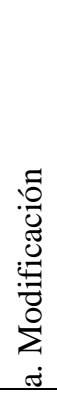 & 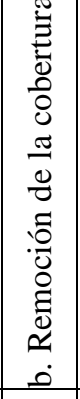 & 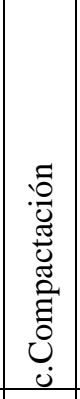 & 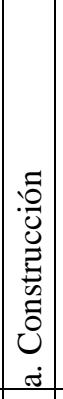 & 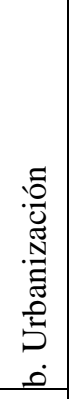 & 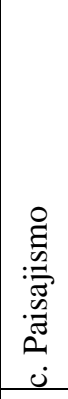 & 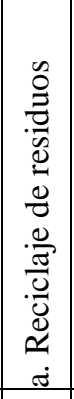 & 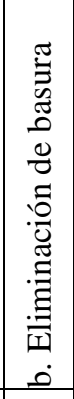 & $\begin{array}{l}\vec{D} \\
\vec{Z} \\
\tilde{n} \\
ن \\
\end{array}$ & 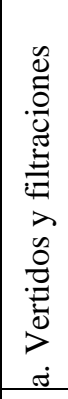 \\
\hline Suelo & -48 & -32 & -20 & -72 & & -32 & 32 & & & $-3,2$ \\
\hline Agua & -21 & & & & -24 & & 24 & & $-2,4$ & $-2,4$ \\
\hline Atmósfera & -54 & -54 & & -27 & & & 21 & & -42 & \\
\hline Flora & -28 & -28 & -36 & -28 & -28 & & 20 & & & \\
\hline Fauna & -21 & -21 & -27 & -21 & -21 & & 15 & & & \\
\hline $\begin{array}{l}\text { Interés } \\
\text { económico y } \\
\text { humano }\end{array}$ & & 63 & & 36 & 81 & & 4,5 & 22,5 & $-3,6$ & \\
\hline $\begin{array}{l}\text { Aspectos } \\
\text { culturales }\end{array}$ & & -32 & & & 72 & -36 & & & & \\
\hline $\begin{array}{l}\text { Relaciones } \\
\text { ecológicas }\end{array}$ & -20 & $-17,5$ & $\begin{array}{c}- \\
17,5\end{array}$ & & -20 & & 12,5 & & & \\
\hline
\end{tabular}

\subsection{Actividades y afección al medio}

La figura 3, muestra las principales actividades e impactos del proyecto. En resumen, se puede apreciar que entre los impactos positivos sobresalen los poco significativos positivos y no significativos positivos, que agrupan el $77 \%$ (10 de los 13 impactos positivos identificados). En el caso de los impactos negativos, se muestran un total de 29 impactos de ellos 25 (86\%), se encuentran entre las categorías no significativo negativo y poco significativo negativo. Existen 3 impactos negativos medianamente significativos y uno significativo negativo. En análisis de las actividades negativas, la modificación de hábitat muestra 6 impactos, 5 la remoción de cobertura y 4 la construcción y compactación.

Mediante el análisis de la matriz de Leopold, se identificaron los impactos negativos y positivos de mayor relevancia. A continuación, se destacan los impactos negativos más importantes:

Modificación de hábitat: Como se puede observar en la figura 3 y tabla 6 , la modificación del hábitat es un impacto negativo, con un grado de afectación considerable, específicamente, la fragmentación de hábitats ha sido uno de 
los principales impactos identificados en la construcción de carreteras Samjang - Sanchung en Korea, donde la evaluación de impacto ecológico permitió identificar los impactos sobre la conservación de la biodiversidad en ecosistemas frágiles.

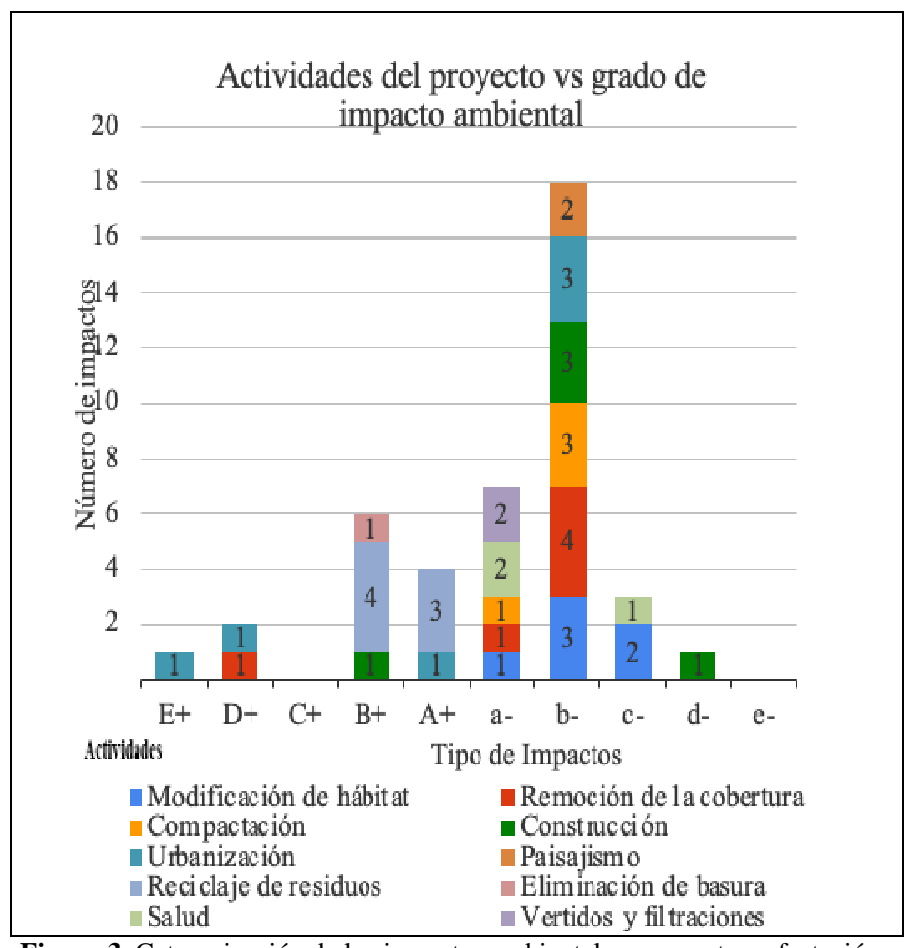

Figura 3. Categorización de los impactos ambientales en cuanto a afectación por actividad

Remoción de la cobertura vegetal del suelo: De igual manera en la figura 3, la remoción de cobertura vegetal del suelo tiene impactos con valores de impacto negativo medianamente significativos. Este valor es comparable con un estudio realizado en Nigeria, donde obtuvieron un valor similar de remoción vegetal, y es frecuente que este tipo de impactos contiene una relevancia moderada en este tipo de proyectos [12]. Según FAO [13] la remoción de la cobertura vegetal es causada por un cambio importante en el uso del suelo a largo plazo. En la zona de estudio se pudo identificar que la remoción de cobertura vegetal provocó pérdidas de ecosistemas y hábitats, especies animales y vegetales, como también la compactación de suelos provocada por la implementación de maquinaria pesada en el momento de la construcción.

Compactación y desertificación del suelo: La compactación es provocada por la maquinaria de construcción que consiste en un proceso artificial en el cual se consigue lograr un aumento de densidad de un suelo natural o relleno [14], provocando así una desertificación debido a que altera la estructura del suelo. En un estudio de impacto ambiental realizado en Nigeria, se obtuvo un impacto poco significativo de la compactación de suelos [12], lo cual generó erosión, pérdida de vegetación y degradación hídrica, de la misma manera que puede afectar la zona de estudio.

Construcción de vías: Como se puede observar en la figura 3, la construcción de vías es un impacto negativo, en este caso es un impacto negativo no significativo. En un estudio de impacto ambiental realizado en Bogotá, obtuvieron un valor similar al del presente estudio. La construcción de vías provoca el levantamiento de polvo y material particulado, afectando la salud de la población con enfermedades respiratorias como son: la bronquitis, neumonía y otras [14]. Dentro de la zona de estudio, las construcciones de vías fueron utilizadas para el transporte de materia prima, escombros, transporte de maquinaria, transporte de vehículos medianos y pequeños, etc.; provocando así el impacto ya mencionado.

Urbanización y viviendas: Como se puede visualizar en la figura 3, este aspecto tiene categoría de impacto negativo no significativo al igual que el estudio realizado en Bogotá, donde el valor de afectación fue de - 9,5\% [14], tomando en cuenta este valor se analizó y está dentro del rango de valoración del proyecto. Esto quiere decir que una urbanización provoca un impacto relevante por la pérdida de cobertura vegetal, modificación de hábitat y la compactación de suelos.

Salud: En esta actividad que es de gran importancia se obtuvo un porcentaje de $-7 \%$ como se puede observar en la figura 3, que fue comparado con un estudio Enshassi, et al. [5], obtuvieron un valor de $-7,2 \%$, abarcando tema importantes para la salud, como es el polvo de cemento, que este contiene metales pesados como el níquel, cobalto, cromo y plomo que pueden provocar enfermedades respiratorias, colocándolo en la categoría de los impactos no significativos negativos, haciendo que este sea un punto muy importante a remediar dentro de los planes de manejo ambiental. Adicionalmente, el polvo generado por las actividades de construcción afecta profundamente la calidad del aire ambiental y podría afectar negativamente la salud de las personas que viven cerca de los sitios de construcción [15]. Por tanto, es necesario considerar para la toma de decisiones el impacto del polvo de la construcción en el medio ambiente circundante.

Paisajismo: Este criterio es un elemento fundamental de las obras o proyectos constructivos, pues los elementos visuales no solo presentan valores estéticos, sino que equilibran las relaciones mutuas entre los valores culturales, económicos y biológicos [16]. Existe una interacción importante entre los seres humanos y la naturaleza, por lo que la evaluación de la calidad visual es importante en la planificación y gestión ambiental. Viloria Villegas, et al. [17] plantea que el medio humano está compuesto por dos sistemas: perceptual, que se refiere a la relación del hombre y su entorno a través del paisaje natural y construido (componente paisaje); y antrópico, que se refiere al hombre en comunidad (componentes políticas, economía y cultura). 
En la evaluación del impacto ambiental (Ver la tabla 6), la modificación o alteración del paisaje puede incidir en el suelo (impacto negativo de -32) y las relaciones culturales (-36). En este último caso, esto está influenciado por la modificación de la calidad visual del paisaje que es producto de características particulares (visibles) del paisaje y como plantea [18] interactúa con procesos psicológicos (perceptuales, cognitivos y emocionales) que son relevantes en el observador humano, por lo que el levantamiento de las viviendas modificará el paisaje y por ende alterará la calidad visual natural del área de estudio. Para compensar estas afectaciones se propone que existan variados espacios verdes, jardines, que puedan integrar los componentes físicos (suelo, agua, aire), y bióticos (la vegetación y la fauna, aunque sean especies menores como aves, insectos). De igual manera, contribuiría a mejorar el aire, reducir el ruido, la erosión y generaría un efecto que regula la humedad y la temperatura.

Vertidos y filtraciones: Se considera como un tipo de impacto negativo no significativo (figura 3 ), provocado por la maquinaria que se encuentra antes, durante y después de la construcción; tiene que ver con el derrame de aceites y lubricantes que se usan para su funcionamiento. Este tipo de impacto es mitigable, dándole un control y mantenimiento apropiado a la maquinaria [14]. La contaminación a las aguas principalmente por la presencia de orgánicos ha sido discutida en $[19,20]$ y puede aumentar los potenciales de acidificación/eutroficación de cursos de aguas superficiales.

Dentro de los impactos positivos analizados los de mayor relevancia son:

Reciclaje de residuos: Es un impacto positivo no significativo, como se puede apreciar en la figura 3 , que disminuye la probabilidad de contaminación por la recuperación de los residuos del proceso. El reciclaje/aprovechamiento de los residuos, puede ser empleado en la obra [21], en otras actividades similares o puede revalorizarse según sea su composición [22], incluso térmicamente como los restos de madera [23].

\subsection{Descripción de los impactos y medidas propuestas más importantes del PMA}

Dentro del proyecto de construcción de viviendas en el Cantón La Maná, parroquia La Maná, se han detectado gran variedad de impactos que de alguna manera u otra afectan a los diferentes tipos de medio que se encuentran cercanos a la construcción. Para poder mitigarlos de una forma adecuada, se hizo énfasis en los principales impactos detectados de cada uno de los planes y cada uno de ellos con su medida correctiva correspondiente, que se detalla más a profundidad en la tabla 7.
Tabla 7. Descripción de los impactos y medidas propuestas más importantes del PMA

\begin{tabular}{|c|c|c|c|}
\hline Plan & $\begin{array}{l}\text { Impactos } \\
\text { detectados }\end{array}$ & Explicación & $\begin{array}{l}\text { Medidas } \\
\text { Propuestas }\end{array}$ \\
\hline \multirow[t]{3}{*}{$\begin{array}{l}\text { Plan de } \\
\text { prevenció } \\
\text { n y } \\
\text { mitigación } \\
\text { de } \\
\text { impactos }\end{array}$} & $\begin{array}{l}\text { Pérdida de } \\
\text { cobertura } \\
\text { vegetal. }\end{array}$ & $\begin{array}{l}\text { Debido a la } \\
\text { remoción } \\
\text { vegetal y la } \\
\text { limpieza del } \\
\text { lugar para la } \\
\text { construcción. }\end{array}$ & $\begin{array}{l}\text { Revegetar en un } \\
\text { lugar cercano o } \\
\text { darle un } \\
\text { segundo uso a la } \\
\text { cobertura } \\
\text { vegetal. }\end{array}$ \\
\hline & $\begin{array}{l}\text { Compactac } \\
\text { ión en el } \\
\text { uso de los } \\
\text { suelos y } \\
\text { desertifica } \\
\text { ción del } \\
\text { suelo. }\end{array}$ & $\begin{array}{l}\text { Debido al } \\
\text { tránsito de la } \\
\text { maquinaria } \\
\text { utilizada en la } \\
\text { construcción. }\end{array}$ & $\begin{array}{l}\text { Realizar el } \\
\text { mantenimiento } \\
\text { de las vías de } \\
\text { acceso interno } \\
\text { por las cuales } \\
\text { transiten las } \\
\text { volquetas con el } \\
\text { trazado de rutas. }\end{array}$ \\
\hline & $\begin{array}{l}\text { Emisiones } \\
\text { de polvo y } \\
\text { material } \\
\text { particulado }\end{array}$ & $\begin{array}{l}\text { El movimiento } \\
\text { de tierra y el } \\
\text { transporte del } \\
\text { material } \\
\text { pétreo, } \\
\text { cemento y } \\
\text { escombros. }\end{array}$ & $\begin{array}{l}\text { Colocar } \\
\text { cobertores de } \\
\text { lona sobre el } \\
\text { material } \\
\text { transportado } \\
\text { para evitar el } \\
\text { esparcimiento. } \\
\text { Regular } \\
\text { volumen el } \\
\text { máximo de } \\
\text { llenado } \\
\text { volquetas hasta } \\
\text { 95\%. } \\
\text { Humedecer } \\
\text { diariamente la } \\
\text { vía de ingreso a } \\
\text { la obra. }\end{array}$ \\
\hline \multirow[t]{2}{*}{$\begin{array}{l}\text { Plan de } \\
\text { Manejo de } \\
\text { Desechos }\end{array}$} & $\begin{array}{l}\text { Daños en } \\
\text { la salud del } \\
\text { personal. }\end{array}$ & $\begin{array}{l}\text { Esto es } \\
\text { generado } \\
\text { debido una } \\
\text { mala } \\
\text { disposición de } \\
\text { los desechos } \\
\text { comunes. }\end{array}$ & $\begin{array}{l}\text { Los desechos } \\
\text { comunes que se } \\
\text { genere por las } \\
\text { actividades } \\
\text { deberán ser } \\
\text { enviados en el } \\
\text { sistema } \\
\text { recolección de } \\
\text { municipio de } \\
\text { Maná. }\end{array}$ \\
\hline & $\begin{array}{l}\text { Cambio en } \\
\text { la calidad } \\
\text { de r los } \\
\text { suelos. }\end{array}$ & $\begin{array}{l}\text { Esto es } \\
\text { generado por } \\
\text { la } \\
\text { incorporación } \\
\text { de materiales } \\
\text { ajenos a la } \\
\text { composición } \\
\text { natural del }\end{array}$ & $\begin{array}{l}\text { Llevar } \\
\text { registro un } \\
\text { adecuado de } \\
\text { todos } \\
\text { desechos } \\
\text { generados, en el } \\
\text { lugar } \\
\text { construcción. }\end{array}$ \\
\hline
\end{tabular}




\begin{tabular}{|c|c|c|c|}
\hline & & suelo. & \\
\hline \multirow[t]{2}{*}{$\begin{array}{l}\text { Plan de } \\
\text { Seguridad } \\
\text { Ocupacion } \\
\text { al y } \\
\text { Seguridad } \\
\text { Industrial }\end{array}$} & $\begin{array}{l}\text { Accidentes } \\
\text { laborales }\end{array}$ & $\begin{array}{l}\text { Debido a que } \\
\text { el personal no } \\
\text { presenta el } \\
\text { EPP adecuado. }\end{array}$ & $\begin{array}{l}\text { Dotar de EPP's } \\
\text { de acuerdo con } \\
\text { las actividades } \\
\text { que realicen los } \\
\text { trabajadores } \\
\text { durante la } \\
\text { ejecución del } \\
\text { proyecto. }\end{array}$ \\
\hline & $\begin{array}{l}\text { Daños en } \\
\text { la salud del } \\
\text { personal. }\end{array}$ & $\begin{array}{l}\text { Accidentes } \\
\text { producidos } \\
\text { dentro del } \\
\text { espacio } \\
\text { laboral. }\end{array}$ & $\begin{array}{l}\text { Disponer de } \\
\text { botiquines de } \\
\text { primeros } \\
\text { auxilios, y estar } \\
\text { preparada para } \\
\text { actuar en casos } \\
\text { de presentarse } \\
\text { emergencias }\end{array}$ \\
\hline $\begin{array}{l}\text { Plan de } \\
\text { Rehabilita } \\
\text { ción de } \\
\text { áreas } \\
\text { afectadas }\end{array}$ & $\begin{array}{l}\text { Modificaci } \\
\text { ón de } \\
\text { caudales. }\end{array}$ & $\begin{array}{l}\text { Se presenta } \\
\text { porque no hay } \\
\text { un adecuado } \\
\text { control de } \\
\text { todos los } \\
\text { materiales de } \\
\text { construcción. }\end{array}$ & $\begin{array}{l}\text { En caso de } \\
\text { alteración de los } \\
\text { cauces de agua } \\
\text { estos deberán } \\
\text { ser dejados en } \\
\text { condiciones } \\
\text { similares a las } \\
\text { iniciales. }\end{array}$ \\
\hline $\begin{array}{l}\text { Plan de } \\
\text { abandono } \\
\text { y entrega }\end{array}$ & \begin{tabular}{ll}
\multicolumn{2}{l}{ Cambios } \\
en el & uso \\
de & los \\
suelos. &
\end{tabular} & $\begin{array}{l}\text { Mala } \\
\text { disposición de } \\
\text { materiales de } \\
\text { construcción. }\end{array}$ & $\begin{array}{l}\text { Notificación al } \\
\text { MAE indicando } \\
\text { que se va a } \\
\text { proceder con el } \\
\text { cierre } \\
\text { abandono del } \\
\text { área. }\end{array}$ \\
\hline \multirow[t]{2}{*}{$\begin{array}{l}\text { Plan de } \\
\text { monitoreo }\end{array}$} & $\begin{array}{l}\text { Cambios } \\
\text { en la } \\
\text { calidad de } \\
\text { los suelos. }\end{array}$ & $\begin{array}{l}\text { Mala } \\
\text { disposición de } \\
\text { materiales de } \\
\text { construcción. }\end{array}$ & $\begin{array}{ll}\text { Retirar todo el } \\
\text { equipo } & \mathrm{y} \\
\text { maquinaria } & \mathrm{de} \\
\text { los frentes } & \mathrm{de} \\
\text { trabajo. } & \end{array}$ \\
\hline & \begin{tabular}{l}
\multicolumn{2}{c}{ Contamina } \\
ción del \\
agua y del \\
aire.
\end{tabular} & $\begin{array}{l}\text { Evaluar la } \\
\text { eficacia y } \\
\text { validez de las } \\
\text { medidas } \\
\text { ambientales } \\
\text { propuestas. }\end{array}$ & $\begin{array}{l}\text { Realizar } \\
\text { monitoreos de } \\
\text { ruido y material } \\
\text { particulado cada } \\
\text { tres meses. }\end{array}$ \\
\hline
\end{tabular}

Para poder mitigar y dar solución a los impactos negativos se planteó una serie de subplanes de manejo ambiental entre ellos están:

Plan de Prevención y Mitigación de Impactos: Es aplicable, pues como menciona Montero-Vega, et al. [11] ayuda a recuperar la cobertura vegetal mediante de la revegetación de áreas afectadas, como se identificaron en la zona de estudio.
Plan de Manejo de Desechos: Es aplicable, debido a que es un impacto positivo, es decir, la mitigación de este será a corto plazo y ayudará a la disminución de desechos provocados por las actividades comunes de la construcción. Sin embargo, en la práctica con la gestión de residuos, solo se enfocan unas pocas medidas, y el manejo de los desechos por parte de las empresas constructoras genera dificultades y plantea desafíos ambientales [1].

Plan de Seguridad Ocupacional y Seguridad Industrial: Este plan es aplicable, tanto en la fase de construcción como en la fase de cierre y abandono, debido a que es necesario en caso de riesgos laborales como lo menciona Galindo Ruiz and Silva Nuñez [14], es esencial para controlar posibles afectaciones a las poblaciones cercanas al área de influencia del proyecto.

Plan de Rehabilitación de Áreas Afectadas: Este plan es aplicable y de gran importancia, ya que va de la mano con el plan de prevención y mitigación para así recuperar las áreas intervenidas y los cauces de agua como lo menciona [11]. En relación con los cauces de agua, deberán ser entregados en condiciones ambientales similares antes del inicio de las operaciones del proyecto, dentro de este plan están las notificaciones respectivas al MAE para dar aviso del cierre y abandono del Proyecto [10].

\section{Conclusiones}

La evaluación del impacto ambiental en todas las fases de un proyecto u obra constructiva es un proceso de gran importancia. Los resultados de este documento revelaron que los principales impactos ambientales son: la contaminación, el uso de los recursos, la destrucción del hábitat y la salud ocupacional, dando como resultado la destrucción de la vegetación, la desertificación de los suelos, la generación de residuos sean estos sólidos o líquidos y la afectación a la salud de los trabajadores. Para mitigar estos impactos, se implementaron planes de manejo ambiental acorde con sus necesidades.

Las distintas acciones del proyecto muestran 25 impactos negativos, poco significativos y no significativos, en cuanto la presencia de los impactos positivos en el componente de interés económico y humano compensa de una forma u otra la aparición de los impactos negativos. El componente ambiental que sufre la mayor parte de afectación es el recurso suelo, por los cambios irreversibles en la extracción de la cobertura vegetal. La modificación o alteración del paisaje muestra dos impactos negativos en el suelo y en las relaciones culturales, por la modificación de la calidad visual del paisaje, lo que denota que la evaluación de la calidad visual es importante en la planificación y gestión ambiental de proyectos de construcción. Se debe llevar un registro de salud y seguridad, como del manejo adecuado de los desechos, para tener un mayor control de los niveles de cumplimiento. Finalmente, se propusieron medidas en los planes de manejo ambiental hacia el entorno físico, biótico y socioeconómico; 
enfocadas a un desarrollo sostenible, que puede contribuir a promover el uso racional de los recursos naturales en los proyectos de desarrollo, especialmente en el sector de la construcción.

\section{REFERENCIAS}

[1] A. Isaksson and H. Linderoth, "Environmental considerations in the Swedish building and construction industry: the role of costs, institutional setting, and information," Journal of Housing and the Built Environment, vol. 33, no. 4, pp. 615632, 2018/12/01 2018.

[2] UNEP. (2016, diciembre/15). The 10YFP programme on sustainable buildings and construction. Available: :http://www.unep.org/10yfp/Programmes/ProgrammeConsultat ionandCurrentStatus/Sustainablebuildingsandconstruction/tabi d/106268/Default.aspx

[3] M. K. Dixit, J. L. Fernández-Solís, S. Lavy, and C. H. Culp, "Identification of parameters for embodied energy measurement: A literature review," Energy and Buildings, vol. 42, no. 8, pp. 1238-1247, 2010/08/01/ 2010.

[4] M. Sandanayake, G. Zhang, and S. Setunge, "A comparative method of air emission impact assessment for building construction activities," Environmental Impact Assessment Review, vol. 68, pp. 1-9, 2018/01/01/ 2018.

[5] A. Enshassi, B. Kochendoerfer, and E. Rizq, "Evaluación de los impactos medioambientales de los proyectos de construcción," Revista ingeniería de construcción, vol. 29, pp. 234-254, 2014.

[6] E. E. V. Aspiazu and L. E. C. Espinoza, "Impactos ambientales producidos por la construcción de vivienda a gran escala en la ciudad de Guayaquil," Dominio de las Ciencias, vol. 3, no. 3, pp. 1066-1085, 2017.

[7] INEC, "Fascículo provincial Cotopaxi," 2010, Available: https://www.ecuadorencifras.gob.ec/wp-

content/descargas/Manu-lateral/Resultados-

provinciales/cotopaxi.pdf.

[8] Senplades, "Plan Nacional de Desarrollo 2017-2021-Toda una Vida," Secretaría Nacional de Planificación y Desarrollo, Quito Ecuador2017, Available: https://www.planificacion.gob.ec/wpcontent/uploads/downloads/2017/10/PNBV-26-OCTFINAL_0K.compressed1.pdf.

[9] N. G. Guallo-Aguinda, L. B. Sarduy-Pereira, E. OrozcoCrespo, and K. Diéguez-Santana, "Las producciones más limpias en el sector textil manufacturero. Un caso de estudio en Tena, Napo, Ecuador," Mikarimin. Revista Científica Multidisciplinaria, vol. 6, pp. 201-218, 2020.

[10] A. I. Soto-Cabrera, A. P. Panimboza-Ojeda, C. G. IlibayGranda, C. R. Valverde-Lara, and K. Diéguez-Santana, "Impacto ambiental de la operación del Centro de faenamiento de la ciudad de Puyo, Pastaza, Ecuador," Prospectiva, vol. 18, no. 1, pp. 60-68, 2020.

[11] F. S. Montero-Vega, C. S. Molina-Cedeño, B. M. PillcoHerrera, L. B. Sarduy-Pereira, and K. Diéguez-Santana, "Evaluación del impacto ambiental de la construcción de una planta de tratamiento de aguas residuales. Caso río Pindo Chico, Puyo, Pastaza, Ecuador," Ciencia, Ambiente y Clima, vol. 3, no. 1, 07/06 2020.

[12] E. A. Ijigah, R. A. Jimoh, B. O. Aruleba, and A. B. Ade, "An assessment of environmental impacts of building construction projects," Civil Environmental Research, vol. 3, no. 1, pp. 93 105, 2013.

[13] FAO, "Evaluación del impacto ambiental. Directrices para los proyectos de campo de la FAO," Organización de las naciones unidas para la alimentación y la agricultura, Roma, Italia2011, Available: http://www.fao.org/3/a-i2802s.pdf.

[14] J. S. Galindo Ruiz and H. D. Silva Nuñez, "Impactos ambientales producidos por el uso de maquinaria en el sector de la construcción," Trabajo de grado del programa de ingeniería civil, Facultad de ingeniería, Universidad Católica de Colombia, Bogotá, Colombia, 2016.

[15] H. Yan et al., "Systematic evaluation framework and empirical study of the impacts of building construction dust on the surrounding environment," Journal of Cleaner Production, p. 122767, 2020/07/18/2020.

[16] Z. Bulut and H. Yilmaz, "Determination of landscape beauties through visual quality assessment method: a case study for Kemaliye (Erzincan/Turkey)," Environmental Monitoring and Assessment, vol. 141, no. 1, pp. 121-129, 2008/06/01 2008.

[17] M. I. Viloria Villegas, L. Cadavid, and G. Awad, "Metodología para evaluación de impacto ambiental de proyectos de infraestructura en Colombia," Ciencia e Ingeniería Neogranadina, vol. 28, no. 2, pp. 121-156, 06/01 2018.

[18] Y. Yao et al., "Assessing the visual quality of green landscaping in rural residential areas: the case of Changzhou, China," Environmental Monitoring and Assessment, vol. 184, no. 2, pp. 951-967, 2012/02/01 2012.

[19] K. Diéguez-Santana, Y. Casas-Ledón, J. A. LoureiroSalabarria, A. Pérez-Martínez, and L. E. Arteaga-Pérez, "A life cycle assessment of bread production: A Cuban case study," Journal of Environmental Accounting and Management, vol. 8, no. 2, pp. 125-137, 2020.

[20] K. Diéguez-Santana, L. E. Arteaga-Pérez, Y. Casas Ledon, and I. L. Rodríguez Rico, "Análisis de ciclo de vida y caracterización ambiental en una industria alimenticia," Revista Centro Azúcar, vol. 40, no. 52-58, 2013.

[21] K. Diéguez-Santana, L. B. Sarduy-Pereira, Y. Casas-Ledón, and L. E. Arteaga-Pérez, "Cleaner Production Implementation in a Cacao Paste Production Plant in Ecuadorian Amazon," Journal of Environmental Accounting and Management, vol. 9, no. 2, pp. 173-188, 2021.

[22] K. Diéguez-Santana, L. B. Sarduy-Pereira, and M. Decker, "Characterization and Quantification of Municipal Solid Waste in Fátima, Ecuadorian Amazon Parish " Journal of Environmental Treatment Techniques, vol. 9, no. 2, pp. 392401, 2021.

[23] L. E. Arteaga-Pérez, C. Segura, and K. D. Santana, "Procesos de torrefacción para valorización de residuos lignocelulósicos. Análisis de posibles tecnologías de aplicación en Sudamérica," Afinidad, vol. 73, no. 573, pp. 60-68, 2016. 